\title{
Escasa presencia de la mujer enfermera en puestos de poder
}

Scarce Presence of Female Nurses in Positions of Power

\section{Alicia Méndez-Salguero, José Eloy García-García}

Servicio de Salud del Principado de Asturias (SESPA)

Contacto: alimensal@gmail.com

Fecha de recepción: 2 de abril de 2019 / Fecha de aceptación: 23 de septiembre de 2019

\section{Resumen}

Introducción: La enfermería es una profesión donde la mayoría de sus componentes son mujeres, sin embargo, los puestos de responsabilidad están mayoritariamente ocupados por hombres enfermeros. El tokenismo explica bien esta realidad.

Objetivos: Valorar si la mujer enfermera sufre discriminación dentro de su propia profesión a nivel sindical y colegial. Comprobar si en los cargos de responsabilidad dentro del ámbito sanitario público, la enfermera sigue estando relegada frente a otros profesionales sanitarios o no.

Metodología: Se realizan búsquedas bibliográficas en diversas bases de datos utilizando las palabras clave y seleccionando con el filtro de diez años artículos en castellano e inglés, y se revisan bases de datos oficiales (INE, EPA, CGE, SATSE y páginas web de los servicios de salud).

Resultados: El 84 \% de la profesión de enfermería son mujeres, aunque en los 15 consejos autonómicos consultados, solo hay 5 mujeres presidentas. En el SATSE, el 58 \% de sus secretarías están ocupadas por mujeres, porcentaje no adecuado con la distribución de género en este colectivo. Así mismo se acusa la no presencia de enfermeras como gerentes de servicios de salud.

Discusión: Se ha demostrado que la enfermería, pese a ser una profesión feminizada, tiene que enfrentarse con el fenómeno del token en su propio mundo, por lo que los hombres dominan las posiciones de poder como así lo evidencian los datos.

Conclusiones: La mujer enfermera sigue estando en posición de inferioridad dentro y fuera de la profesión.

Palabras clave: enfermera, tokenismo, escalera de cristal, feminización.

\footnotetext{
Abstract

Introduction: It's a fact that nursing is a profession where most of their components are women, nevertheless, responsibility and decision-making positions are mainly occupied by male nurses, and this is like this in spite of that they only represent $16 \%$ of the professional contingent. The tokenism explains well this reality.

Objectives: To value if the female nurse suffers discrimination inside the own profession, in the nurses' union and in the professional association. To verify if in the responsibility positions inside the Regional Health Services, the nurse is still relegated to other health professionals or not.

Methodology: Bibliography researches were made in various databases using the keywords and selecting with the ten years filter articles in Spanish and English, and official databases were revised (INE, EPA, CGE, SATSE and websites of the Regional Health Services).

Results: The $84 \%$ of the professionals nurses are females, although in the 15 Regional Nurses Councils consulted, there are only 5 chairwomen. In the SATSE, $58 \%$ of secretariats are occupied by women, percentage that is not appropriate with the gender distribution in this collective. It is also alleged that nurses are not present as managers of Health Services.

Discussion: It has been shown that nursing, despite being a feminized profession, has to deal with the phenomenon of the token in their own world so men dominate positions of power as evidenced by the data.

Conclusions: The female nurse is still in an inferior position both inside and outside the profession.
} 


\section{Introducción}

\section{Enfermería, mujer y sociedad}

El análisis de la transición histórica de la enfermería a lo largo de los últimos cien años permite entender muchas de las situaciones que está viviendo el colectivo, como la escasa visibilidad y el bajo nivel de reconocimiento propio y ajeno ${ }^{1}$. A día de hoy, aún comparten espacio profesional enfermeras con diferentes titulaciones como es el caso de las ATS, DUE y graduadas de Enfermería; no obstante, durante el pasado siglo también hubo instructoras sanitarias y practicantes. Esta diferencia de titulaciones se corresponde con momentos muy distintos en la profesión, y se resuelve con la denominación común de "enfermeras"”2.

El plan Bolonia ha contribuido positivamente a la superación de estas nomenclaturas; además, el término enfermera es aceptado hoy tanto por el CIE como por el Consejo General de Enfermería de España, o instituciones internacionales como la OMS. Si bien la diplomatura supuso la entrada en la universidad, el acceso a estudios de grado y postgrado junto con la posibilidad de especializarse vía residencia, ha colocado a la enfermería al mismo nivel de cualquier otra profesión universitaria. Ello ha supuesto un evidente avance académico, facultando definitivamente a las enfermeras para la investigación y la docencia, aunque la profesión sigue adoleciendo de viejos problemas como la escasa visibilidad, a lo cual contribuyen hechos como que las escuelas de Enfermería han pasado mayoritariamente a estar integradas en las facultades de Ciencias de la Salud, en las que se engloban otras disciplinas como la fisioterapia, la podología, la medicina...

El gran problema que arrastra la enfermería, al ser una profesión eminentemente femenina ${ }^{3}$, es paralelo al de la mujer a lo largo de la historia, ya que ambas han sido relegadas a un segundo nivel frente a la supuesta supremacía masculina. Dicha realidad se ha sostenido a pesar de que se van eliminando progresivamente todos los mitos sobre la inferioridad de la mujer, y a pesar también de la superación de la vieja creencia de que la mujer ha sido la causa de todos los problemas de la sociedad ${ }^{4,5}$. Las tareas propias e independientes de la enfermera: acompañar, consolar, convencer..., englobadas en el concepto de cuidado, a la vista de la sociedad, parecen algo más femenino y doméstico que el desempeño de tareas más técnicas o que implican manejo de aparataje, tecnología, etc., lo cual se asocia con lo masculino ${ }^{6}$.
Hoy la enfermería trata de hacerse visible mediante campañas en redes sociales, webs de sindicatos y blogs sanitarios $^{7-9}$, que, entre otros medios, intentan cambiar la imagen desajustada que las enfermeras proyectan hacia afuera. Y es que las enfermeras no han sabido transmitir lo qué significa "ser enfermera". En este sentido, ni el cine ${ }^{10}$ ni los medios de comunicación como la prensa escrita o la televisión ${ }^{11-12}$ han ayudado; de hecho, diversos estudios demuestran que gran parte de la sociedad aún las conoce como las "ayudantes del médico" ". Además, la enfermería sigue siendo valorada como una profesión de mujeres cuya característica más significativa es la sumisión y la dependencia, sin un campo propio, sin apenas formación o, sin ir más lejos, una profesión llena de estereotipos sexuales ${ }^{14-16}$.

\section{El “tokenismo” y su relación con la enfermería}

Cuando revisamos todos estos argumentos surge una cuestión importante: siendo la enfermería una profesión tradicionalmente de mujeres, ya que en 2017 representan el 84,33\% de la enfermería colegiada en España $^{17}$, ¿por qué los dirigentes y las caras visibles de la profesión, suelen ser hombres? La respuesta a esta pregunta viene siendo objeto de estudio e investigación desde hace años, dado que no es una circunstancia exclusiva de la enfermería, sino que afecta a muchas de las profesiones compuestas mayoritariamente por mujeres.

La teoría de Kanter del tokenismo, de $1977^{18}$, explica que en los grupos humanos dentro del mundo profesional o empresarial en los que hay desigualdad en el número relativo a un género, el grupo minoritario es considerado como token. Pertenecer al grupo token tiene unas consecuencias descritas por Kanter, como la alta visibilidad (aumenta la presión social), o la polarización del grupo (todo lo dicho por ellos es tomado como algo importante, aunque no lo sea). Además, las características del token deben coincidir con los estereotipos de los grupos a los que pertenecen (género, raza, condición sexual...), lo que implica la asunción del rol, que, en el caso que nos ocupa, será el rol de género $^{19}$. Se podría entender tokenismo como una forma de incluir simbólicamente a esa minoría en el grupo permitiéndosele alguna ventaja o concesión, pero esta supuesta ventaja no siempre es real, ya que detrás de determinadas situaciones que pueden parecer ventajosas, se esconden prejuicios que finalmente generan una posición de inferioridad para dicha minoría. A juicio de la autora este fenómeno ocurriría tanto entre hombres como entre mujeres, pero dicha afirma- 
ción se ha debatido ampliamente, ya que no es exactamente igual para ambos, o dicho de otra manera, el tokenismo puede ser positivo o negativo para el token según sea mujer u hombre ${ }^{20}$. Si nos fijamos en el caso de la enfermería o de las profesiones donde los "token" son los hombres, diversos estudios ${ }^{21-24}$ han descrito que los varones no tienen obstáculos serios en sus carreras gracias a su "capital de género". Este concepto implica que las características asociadas a lo masculino a menudo actúan como un beneficio para los hombres en las profesiones feminizadas $^{25}$. Si lo relacionamos con la enfermería, algunos estudios ${ }^{6}$ apuntan que, para superar toda esta problemática, la profesión debe desprenderse de su simbología femenina, se deben abordar proyectos de innovación y conseguir el apoyo institucional global.

Más allá del concepto que acabamos de desarrollar, pero siguiendo en la misma línea de estudio, nos encontramos con lo que Christine Williams denominó la "escalera de cristal",26. Dicho constructo nos ayuda a entender la situación descrita anteriormente en la enfermería: Williams considera que los efectos del sexismo machista aumentan los posibles beneficios del tokenismo en los hombres, ya que son objeto de trato no discriminatorio o incluso preferencial tanto en la contratación como en las situaciones de ascenso, algo que está ampliamente aceptado en la cultura del trabajo y fue denominado como la escalera de cristal. Según esta visión los hombres mantienen las ventajas asociadas a su estatus de género cuando están en desventaja numérica, y por lo tanto acceden a las posiciones más beneficiosas o puestos de poder a través de estas escaleras de cristal, en donde no hay traspiés ni obstáculos importantes como las mujeres tendrían. En el caso de ellas o de otros grupos con menor estatus, se toparían con un techo de cristal $^{27}$, es decir, con todas esas barreras que les impiden seguir subiendo. Los hombres se benefician de su posición token ya que se asume que tienen entre otros valores añadidos, una mayor capacidad de liderazgo y una actitud mejor hacia el desarrollo de la práctica profesional, por lo que ascienden más rápido que sus compañeras y tienden a monopolizar las posiciones de poder, obteniendo más beneficios que perjuicios (mayor visibilidad, progreso más rápido en su carrera, mejores sueldos... $)^{28}$. La autonomía, el poder y la profesionalidad son entendidos como rasgos masculinos, que en las profesiones mayoritarias de mujeres escasean ${ }^{6}$. Esta diferencia en el tokenismo se explica añadiendo factores socioculturales a la posición o estatus de la persona o grupo que está en minoría, en este caso, hombres por encima de mujeres, o mujeres con poder adquisitivo o posición social elevada por encima de otras mujeres de inferior clase social.

Cuando se hace un análisis de género en las sociedades cientificas, se nota que el patriarcado y la jerarquía van de la mano. ${ }^{29}$

\section{Objetivos}

Primeramente, se pretende valorar si la mujer enfermera sufre discriminación dentro de su propia profesión a nivel sindical y colegial, es decir, si se guarda la proporción adecuada entre hombres y mujeres en los puestos de representación al frente del sindicato mayoritario de enfermeras y de los colegios profesionales.

En segundo lugar, comprobar si en los cargos de responsabilidad dentro del ámbito sanitario público, la enfermera sigue estando relegada frente a otros profesionales, sanitarios o no.

\section{Material y método}

Se realizan búsquedas bibliográficas en las bases de datos Dialnet, Cuiden, Pubmed, y Google Scholar, utilizando los términos "tokenism", "glass escalator", “enfermería AND techo de cristal”, "enfermería AND tokenismo", "feminización AND enfermería", "feminización AND salud”. Se seleccionan artículos publicados en inglés y español con un filtro de 10 años, y posteriormente se desarrolla una búsqueda consecutiva de las diferentes bibliografías que aportan información fundamental. Se obtienen datos concretos y actualizados de diferentes colectivos a través de las bases de datos oficiales que permiten hacer las comparaciones pertinentes.

1. INE. Se extrae la información del Instituto Nacional de Estadística sobre la colegiación de enfermeras en España en el año 2017. También se utilizan los datos presentados en el INE para comparar el porcentaje de mujeres en las diferentes profesiones sanitarias.

2. EPA. Se extraen datos de la Encuesta de Población Activa de profesionales sanitarios, realizada en el último trimestre del 2018.

3. Se recogen datos extraídos de la página del Consejo General de Enfermería, de donde se obtiene el organigrama del Consejo y también de los diferentes Colegios Interprovinciales. 
4. Se consultan los datos de las 17 CC.AA. de España sobre los directores gerentes de cada uno de los servicios de salud.

5. Se obtienen los datos del sindicato mayoritario de enfermería, SATSE, tanto a nivel nacional como a nivel interprovincial.

Se realiza una revisión de los datos obtenidos a nivel cualitativo y nominal, extrayendo la información necesaria relacionada con los objetivos propuestos.

\section{Resultados}

1. Representación cuantitativa de la mujer en los colegios profesionales de enfermería y en el sindicato mayoritario de enfermeras.

Según los datos del INE de 2017, la colegiación de enfermeras en España es de 299824 personas. De ellas, 252845 son mujeres, lo que hace un total de $84,33 \%$ frente al 15,67\% de hombres. Este es el mayor porcentaje de mujeres dentro de todas las profesiones sanitarias, y por ello a lo largo del artículo se habla en femenino tal y como ha sido aceptado por el Consejo Internacional de Enfermería $(\mathrm{CIE})^{2}$ y otras instituciones nacionales e internacionales, de tal forma que el término enfermera engloba tanto a las mujeres como a los hombres que ejercen dicha profesión.

Podemos afirmar entonces que, en una profesión donde más del $84 \%$ de las integrantes son mujeres, debería guardarse la proporción pertinente respecto a su presencia en cargos de poder tanto en el ámbito de los colegios profesionales como en el de los sindicatos. Por ello, en dichas organizaciones, por cada 10 representantes 2 deberían ser hombres y 8 deberían ser mujeres aproximadamente.

El análisis de estas proporciones aporta la siguiente información:

\section{1. Órganos colegiales}

En primer lugar, tenemos que mencionar al Consejo General de Colegios Oficiales de Enfermería de España, siendo este el referente de la profesión enfermera en el territorio nacional. Es el órgano máximo de representación de la enfermería y se divide en 17 consejos autonómicos y 52 colegios provinciales.

A pesar de no disponer de información de los colegios de 2 CC.AA. debido a los procesos que están ocurriendo en ellas, en los otros 15 consejos autonómicos hay 10 hombres y 5 mujeres. Esto hace que el $67 \%$ de los representantes de los colegios autonómicos de los que tenemos datos sean hombres, a pesar de que apenas constituyen el 16\% del total de la colegiación.

En la misma página web del Consejo General, con sus 52 delegaciones provinciales, podemos encontrar la distribución de los presidentes/presidentas de las comunidades autónomas que solamente tienen una provincia, habiendo en ellas un $58 \%$ de hombres frente

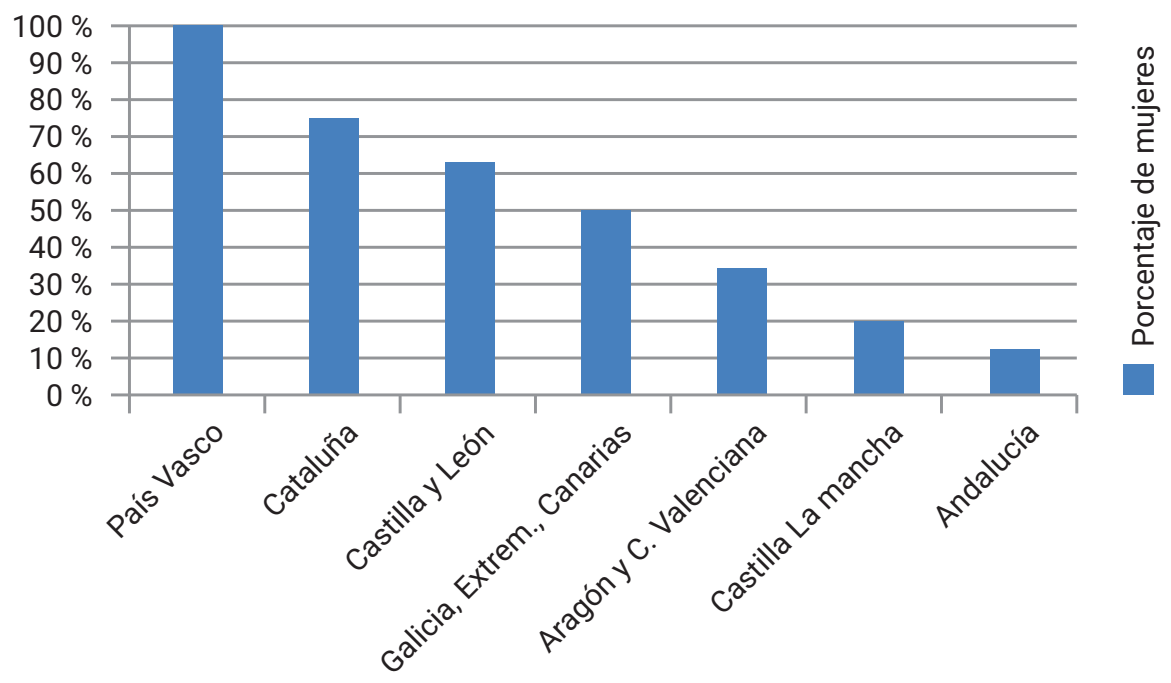

Gráfico 1. Mujeres presidentas en CC. AA. pluriprovinciales 
al $42 \%$ de mujeres presidentas de colegios de enfermería.

En lo relativo al análisis de los datos de los colegios de todas las comunidades autónomas con más de una provincia (Gráfico 1), se muestra el porcentaje de mujeres presidentas en cada una de las CC.AA. pluriprovinciales. Los datos más positivos se corresponden con el País Vasco, donde sus tres presidencias las ocupan mujeres, y el dato más negativo en Andalucía, teniendo en cuenta que solamente hay una mujer presidenta en una comunidad autónoma compuesta por 8 provincias. Cabe destacar que solamente País Vasco tiene un $100 \%$ de mujeres en todas sus provincias, siendo el promedio de mujeres presidentas de los datos obtenidos el $51 \%$, lo que está muy lejos de corresponderse con el porcentaje de mujeres colegiadas.

Por tanto, se cierra este epígrafe constatando que son hombres tanto el presidente del Consejo General, como el $67 \%$ de los representantes autonómicos de los que tenemos datos. El $58 \%$ de los presidentes de las CC.AA. uniprovinciales son hombres, y los porcentajes de hombres al frente de los colegios de las CC.AA. pluriprovinciales siguen siendo mucho mayores de lo esperado, encontrándose una media del $49 \%$ en dichos puestos cuando en el colectivo solo representan algo menos del $16 \%$ del total.

\begin{tabular}{|c|c|c|c|c|c|c|}
\hline SATSE & S ECRETARIA/O & $\begin{array}{c}\text { ALTOS } \\
\text { CARGOS } \\
\text { MUJER }\end{array}$ & $\begin{array}{c}\text { ALTOS } \\
\text { CARGOS } \\
\text { HOMBRES }\end{array}$ & $\begin{array}{c}\text { VOCALES } \\
\text { MUJER }\end{array}$ & $\begin{array}{l}\text { VOCALES } \\
\text { HOMBRE }\end{array}$ & $\begin{array}{c}\text { TOTAL } \\
\text { PERSONAL } \\
\text { (sin secretaría) }\end{array}$ \\
\hline ANDALUCÍA & HOMBRE & 0 & 3 & 0 & 0 & 3 \\
\hline ARAGÓN & MUJER & 1 & 0 & 0 & 0 & 1 \\
\hline AS TURIAS & MUJER & 4 & 0 & 5 & 4 & 13 \\
\hline CANARIAS & HOMBRE & 2 & 0 & 0 & 0 & 2 \\
\hline CANTABRIA & MUJER & 2 & 0 & 4 & 1 & 7 \\
\hline CAS TILLA LA MANCHA & HOMBRE & 2 & 0 & 0 & 0 & 2 \\
\hline CAS TILLA Y LEÓN & MUJER & 3 & 0 & 0 & 0 & 3 \\
\hline CATALUNYA & MUJER & 5 & 2 & 0 & 0 & 7 \\
\hline CEUTA & MUJER & 1 & 2 & 0 & 0 & 3 \\
\hline C. VALENCIANA & MUJER & 1 & 0 & 0 & 0 & 1 \\
\hline EUS KADI & HOMBRE & 2 & 1 & 0 & 0 & 3 \\
\hline EXTREMADURA & HOMBRE & 1 & 3 & 0 & 0 & 4 \\
\hline GALICIA & MUJER & 2 & 0 & 0 & 0 & 2 \\
\hline ILLES BALEARS & HOMBRE & 0 & 1 & 0 & 0 & 1 \\
\hline LA RIOJA & MUJER & 1 & 0 & 3 & 1 & 5 \\
\hline MADRID & MUJER & 2 & 1 & 0 & 0 & 3 \\
\hline MELILLA & HOMBRE & 1 & 0 & 0 & 0 & 1 \\
\hline NAVARRA & MUJER & 1 & 0 & 0 & 0 & 1 \\
\hline REGIÓN DE MURCIA & HOMBRE & 0 & 3 & 0 & 0 & 3 \\
\hline
\end{tabular}

Tabla 1. Secretarios y cargos provinciales del SATSE 


\subsection{Sindicatos}

El mundo sindical para la enfermería se divide en dos vertientes: el sindicato mayoritario y exclusivo de enfermeras que es el SATSE, y las ramas sanitarias o federaciones de otros sindicatos que funcionan con menor número de afiliados, y que también están abiertos a otros profesionales del entorno sanitarios o no.

Cabe destacar que el SATSE, además de unas siglas poco actualizadas, tiene como presidente a un hombre, algo que redunda en la brecha que nos ocupa. Pero si se obtienen datos de las diferentes delegaciones provinciales, con las secretarías correspondientes, podemos comprobar que las mujeres superan a los hombres en las secretarías ( $58 \%$ vs. $42 \%$ ). Sobre las secretarías y altos cargos provinciales, se ha elaborado la Tabla 1 en la que se plasman los altos cargos, los vocales y la secretaría, y se ha comprobado lo siguiente:

- De 19 delegaciones provinciales, 8 son dirigidas por hombres, lo que supone que el $42 \%$ del total de las Delegaciones tienen hombres al frente.

- En los siguientes 3 casos, SATSE Andalucía, Illes Balears y Región de Murcia, todo su personal son hombres (incluido el secretario); es decir, en el 16\% de las delegaciones de este sindicato, son todo hombres.
- Existen 2 casos, SATSE Ceuta y Extremadura, en el que los hombres duplican o triplican, respectivamente, a las mujeres, situación que representa el $11 \%$ del total de las delegaciones.

- Existen 4 casos en el que todo el personal es mujer (SATSE Aragón, Castilla y León, Comunidad Valenciana y Navarra), que son el $21 \%$ del total de las delegaciones.

\section{La mujer, la enfermería y la falta de representación en la Sanidad Pública.}

Según la Encuesta de Población Activa (EPA) desarrollada por el INE en España, con datos del último trimestre de $2018^{30}$, las mujeres ocupan el $71,7 \%$ de los puestos de profesionales de la salud, lo cual deja claro el nivel de feminización del sector.

En la Tabla 2 se recogen, según los datos del INE, la cantidad de profesionales sanitarios colegiados a $31 \mathrm{de}$ diciembre del 2017 en orden de mayor a menor número. Aquí podemos comprobar que la profesión con mayor representación numérica es la enfermería, con casi 300000 profesionales, lo que hace un porcentaje del $36,5 \%$ del total. El siguiente colectivo es el médico, con una representación cercana al $31 \%$, quedando el resto de las profesiones muy alejadas de las cifras de estas dos primeras.

\begin{tabular}{|c|c|c|}
\hline $\begin{array}{c}\text { Profesionales sanitarios colegiados } \\
\text { a 31 de diciembre 2017 }\end{array}$ & $\mathbf{N}^{\mathbf{0}}$ & $\mathbf{\%}$ \\
\hline Enfermeras & 299824 & 36,5 \\
\hline Médicos & 253796 & 30,9 \\
\hline Farmacéuticos & 72500 & 8,83 \\
\hline Fisioterapeutas & 51130 & 6,22 \\
\hline Dentistas & 36689 & 4,47 \\
\hline Veterinarios & 32555 & 3,96 \\
\hline Psicólogos & 25857 & 3,15 \\
\hline Ópticos-Optometristas & 17372 & 2,11 \\
\hline Logopedas & 8971 & 1,09 \\
\hline Podólogos & 7406 & 0,9 \\
\hline Protésicos dentales & 7248 & 0,88 \\
\hline Dietitas nutricionistas & 4086 & 0,5 \\
\hline Terapeutas ocupacionales & 3502 & 0,43 \\
\hline Químicos & 323 & 0,04 \\
\hline Físicos & 189 & 0,02 \\
\hline TOTAL & $\mathbf{8 2 1} \mathbf{4 4 8}$ & $\mathbf{1 0 0}$ \\
\hline
\end{tabular}

Tabla 2. Profesionales sanitarios colegiados a 31 de diciembre 2017 
Posteriormente se consultan los datos de las direcciones de gerencia de los servicios de salud de las comunidades autónomas que se presentan a continuación. En primer lugar, destacar que solamente 3 de las gerencias están ocupadas por mujeres, pese a que del total de trabajadores/trabajadoras más del $71 \%$ son mujeres.

Igualmente llamativo es que la enfermería ni siquiera aparece entre las profesiones de los gerentes, como muestra el Gráfico 2. A fecha de la revisión realizada con datos de marzo del 2019, la mayoría de los gerentes son médicos, el $76 \%$, de entre los cuales hay una mujer. El 12\% son titulados en Derecho, entre ellos también una mujer. De los dos gerentes restantes, uno es titulado en Ciencias Económicas, varón también, y la otra es farmacéutica, titulación que ocupa el tercer puesto en el ranking de profesiones sanitarias por número de colegiados trabajando en el sector sanitario.

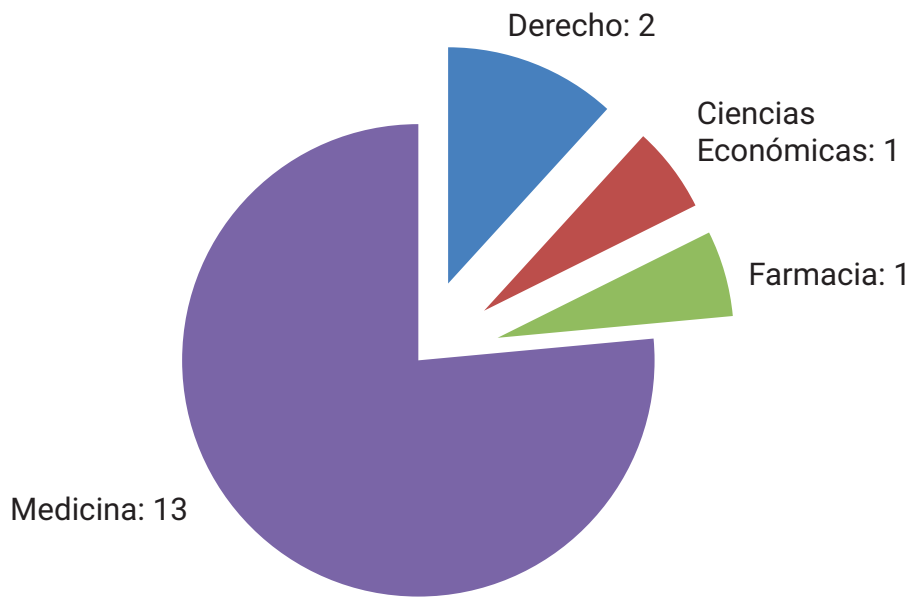

Gráfico 2. Profesiones gerentes Sanidad

\section{Discusión}

Se ha demostrado que la enfermería, pese a ser una profesión en la que más del $84 \%$ de sus componentes son mujeres, tiene que enfrentarse con el fenómeno del token en su propio mundo, por lo que los hombres dominan las posiciones de poder como así lo evidencian los datos. El presidente del Consejo General de Colegios de Enfermería es un hombre, el $67 \%$ de los presidentes de los colegios provinciales de los que se tienen datos también son hombres, y el $58 \%$ de los presidentes de los colegios de enfermería de las CC.AA. también lo son. El fenómeno de la escasez de mujeres en los órganos de dirección de los colegios profesionales es recogido en otros estudios ${ }^{31}$, en donde se destaca que tanto en Medicina como en otras titulaciones: Fisioterapia, Podología, Odontología... sigue manteniéndose esta situación, siendo estas diferencias menos abultadas en Farmacia, Psicología y Enfermería. Lo mismo sucede en el área sindical, en donde el presidente del sindicato mayoritario de enfermeras es un hombre y en donde las delegaciones de dicho sindicato acusan una desproporción negativa en lo que a la presencia de las mujeres se refiere. A su vez, dentro de la Administración Pública, tampoco se ha desarrollado sensibilidad suficiente respecto a la brecha que hay en la ocupación de puestos de responsabilidad, como corroboran las cifras sobre hombres gerentes/mujeres gerentes de los servicios de salud, teniendo en cuenta además que las mujeres representan la inmensa mayoría de las personas que trabajan en la sanidad. Colomer y Peiró ${ }^{32}$, explican que el techo de cristal está en relación directa con factores estructurales propios de la sociedad patriarcal, en donde, a pesar de las leyes paritarias, las mujeres tienen más difícil acceder a dichos espacios. Así mismo, hablan del efecto cohorte que se da por la tardía incorporación de la mujer a estos espacios. En todo esto juega un papel esencial la visión androcéntrica del mundo que relaciona a la mujer con el trabajo reproductivo, que es invisible socialmente, 
no remunerado y no proporciona autonomía personal. El hombre, por el contrario, está al lado del trabajo productivo, visible, bien valorado y que le permite ser autónomo ${ }^{33}$.

\section{Conclusiones}

Tras la revisión de todos los datos adjuntos, se puede concluir que la mujer enfermera sigue estando en una posición de inferioridad tanto dentro como fuera de la profesión. Dentro, la mujer se ve relegada de los cargos de responsabilidad tanto en colegios profesionales como en el sindicato mayoritario de enfermeras, donde, pese a que hay más mujeres que hombres, siguen siendo unos porcentajes que no se ajustan a la realidad numérica de las mujeres en la enfermería. Fuera de la misma, en relación con el resto de profesiones sanitarias, se constata que otras titulaciones ocupan de forma mayoritaria los puestos de poder y toma de decisiones.

Es por ello que se considera la existencia de una doble discriminación que afecta a la mujer y a la enfermera en la sociedad actual. A pesar de que la mujer representa a nivel mundial aproximadamente las tres cuartas partes del colectivo de profesionales de la sanidad, su presencia en puestos de responsabilidad y visibilidad se mantiene mermada en virtud de la cultura machista que impera en nuestro sistema. Es una evidencia de la situación de discriminación intraprofesional que, en un colectivo donde más del $84 \%$ son mujeres, el presidente del Consejo General es un hombre, al igual que lo ha sido su antecesor, pero el problema se hace aún mayor cuando analizamos el resto de los datos ofrecidos por el mismo Consejo General, lo que quiere decir que las proporciones siguen sin cumplirse en los organismos más representativos de nuestra profesión. Por otra parte, las enfermeras son las profesionales dentro de nuestro sistema sanitario más numerosas, pero cuya titulación sigue sin estar presente o lo hace de forma escasísima en la nómina de gerentes de servicios de salud, consejerías de sanidad, gerencias de área, u otros puestos que se adjudican a profesionales con otras titulaciones, sanitarias o no.

Sería conveniente esclarecer las causas por las que las mujeres enfermeras siguen soportando un rol secundario dentro de su propia profesión, tanto en el ámbito colegial como en el sindical, frente a los hombres. De la misma forma, también sería necesario aportar luz acerca de la falta de enfermeras en los puestos de responsabilidad del Sistema Nacional de Salud.

\section{Limitaciones del estudio}

Se ha realizado un estudio descriptivo transversal de la situación actual de la enfermería, partiendo de la base de que los cambios políticos más recientes ocurridos en los diferentes servicios de salud necesariamente están produciendo alguna modificación de los datos aquí registrados. Además, se precisaría desarrollar un diseño longitudinal para poder valorar si el problema a estudio mejora o se agrava con el tiempo.

\section{Bibliografía}

1. Torres A, Sanhueza O. Desarrollo de la autoestima profesional en enfermería. Invest educ enferm 2006; (24)2: 112-119. 2006;112-9.

2. CIE. Definiciones | ICN - International Council of Nurses [Internet]. [cited 2019 Feb 16]. Available from: https://www.icn.ch/es/politica-de-enfermeria/definiciones

3. González J. Enfermería y feminismo (I). Salud a Diario [Internet]. 2018 Jun; Available from: https://www.saludadiario.es/opinion/enfermeria-y-feminismo-i

4. Blanco Corujo O. La polémica feminista en la España ilustrada ALMUD, editor. Oviedo; 2010.174p.

5. Moi T. Teoría literaria feminista. Cátedra. 1985. $200 \mathrm{p}$

6. Celma M, Ángel V, Delgado A. INFLUENCIA DE LA FEMINIZACIÓN DE LA ENFERMERÍA EN SU DESARROLLO PROFESIONAL. Antropol Exp [Internet]. 2009;9:119-36. Available from: http://revista.ujaen.es/rae

7. SATSE. \#EnfermeríaVisible [Internet]. Soy Enfermera. 2018 [cited 2019 Feb 16]. Available from: https://www.soyenfermera.es/enfermeria-visible/

8. Santillán A. Manifiesto por una enfermeria visible [Internet]. Ebevidencia. 2014 [cited 2019 Feb 16]. Available from: https://ebevidencia.com/archivos/2921

9. Fernández S. Enfermería visible [Internet]. Cuidando.es. 2014 [cited 2019 Feb 16]. Available from: https://www.cuidando.es/etiqueta/enfermeria-visible/

10. Almansa P. Enfermería y cine posfranquista. Una visión frívola. Enfermería Glob. 2004;4:1-8.

11. Marchador Pinillos $B$. Influencia de la prensa escrita en la imagen de la profesión enfermera. Bibl Lascasas. 2005;46(1):185-9.

12. Ponce-Blandón JA, Romero-Martín M, López-Verdugo M, LópezNarbona J. La imagen de la enfermería en los medios de comunicación. In: I Jornadas Nacionales de Comunicación en Salud IV Jornadas de Comunicación en Salud. 2016. p. 1.

13. Errasti-lbarrondo B, Arantzamendi-Solabarrieta M, Canga-Armayor N. La imagen social de la enfermería: una profesión a conocer. An Sist Sanit Navar [Internet]. 2012 Aug [cited 2019 Sep 18];35(2):269-83. Available from:

http://scielo.isciii.es/scielo.php?script=sci_arttext\&pid=S1137$66272012000200009 \&$ lng=en\&nrm=iso\&tlng=en

14. Domínguez Alcón C. La imagen de la enfermera en la sociedad. Rol Enfermería. 1985;(83):66-72.

15. Salazar S, Morcillo A. Comunicación, imagen social y visibilidad de los Cuidados de Enfermería. ENE, Rev Enfermería [Internet]. $2013 ; 7(1)$. Available from: https://eneenfermeria.org/ojs/index.php/ENE/article/view/256

16. Rodríguez-Álvaro M, Brito-Brito PR. \# 24h24p : Imagen e Identidad. ENE, Rev Enfermería. 2013;7(3):3-5.

17. INE. Enfermeros colegiados por tipo de especialidad, año y sexo. [Internet]. Instituto Nacional de Estadística. 2017. Available from: https://www.ine.es/jaxi/Datos.htm?path=/t15/p416/serie/I0/\& file $=$ s08001.px 
18. Kanter RM. Men and Women of the Corporation. Basic Books, INC. New York; 1977. 390 p.

19. Heikes EJ. When Men Are the Minority: The Case of Men in Nursing [Internet]. Vol. 32, The Sociological Quarterly. Taylor \& Francis, Ltd.; [cited 2019 Mar 6]. p. 389-401. Available from: https://www.jstor.org/stable/4120914

20. Cohen LL, Swim JK. The Differential Impact of Gender Ratios on Women and Men: Tokenism, Self-Confidence, and Expectations. Personal Soc Psychol Bull [Internet]. 1995 Sep 2 [cited 2019 Mar 6];21(9):876-84. Available from: http://journals.sagepub.com/doi/10.1177/0146167295219001

21. Bielby DD, Williams CL. Still a Man's World: Men Who Do "Women's Work". Contemp Sociol [Internet]. 1995 Nov [cited 2019 Mar 6];24(6):809. Available from: http://www.jstor.org/stable/2076713?origin=crossref

22. Floge L, Merrill DM. Tokenism reconsidered: Male nurses and female physicians in a hospital setting. Soc Forces. 1986;64(4):925-47.

23. Cross S. "Girls" jobs for the boys? Men, masculinity and nontraditional occupations. Gender, Work Organ. 2002;9(2):204-26.

24. Crocker J, McGraw K. What's good for the goose, is not good for the gander. Am Behav Sci. 1984;27(3):357-69.

25. McDowell J. Masculinity and Non-Traditional Occupations: Men's Talk in Women's Work. Gender, Work Organ [Internet]. 2015 May [cited 2019 Mar 6];22(3):273-91. Available from: http://doi.wiley.com/10.1111/gwao.12078

26. Williams CL. The Glass Escalator: Hidden Advantages for Men in the "Female" Professions. Soc Probl. 1992;39(3):253-67.

27. E J. La "escalera de cristal" y la neutralidad de género. Expansion. 2018;

28. Simpson R. Masculinity at work: The experiences of men in female dominated occupations. Work Employ Soc. 2004;18(2):349-68.

29. Casado-Mejía R, Botello-Hermosa A. Representatividad de las mujeres en las sociedades científicas españolas del ámbito de la salud en 2014. Gac Sanit [Internet]. 2015;29(3):209-12. Available from: http://dx.doi.org/10.1016/j.gaceta.2014.09.010

30. INE. Escuela de Población Activa - Profesionales de la Salud [Internet]. 2018. Available from: https://www.ine.es/jaxiT3/Datos.htm?t=4077

31. Botello Hermosa A, Casado Mejía R, Germán Bes C. PRESENCIA DE LAS MUJERES EN LOS ÓRGANOS DE DIRECCIÓN DE LOS COLEGIOS PROFESIONALES DEL ÁMBITO DE LA SALUD EN 2015. Rev Esp Salud Pública [Internet]. 2015;89(6):1-6. Available from:

https://dialnet.unirioja.es/servlet/articulo?codigo=5310365

32. Colomer Revuelta C, Peiró Pérez R. ¿Techos de cristal y escaleras resbaladizas? Desigualdades de género y estrategias de cambio en SESPAS. Gac Sanit. 2002;16(4):358-60.

33. Escribano Martínez MÁ, Picazo Lahiguera C, Gamero Vázquez N, Lasanta Berdejo E. La feminización de la sanidad y el techo de cristal: el caso de la Comunidad Valenciana. Enfermería Integr. 2010;(89):32-6. 